Article

\title{
Near-Field Coupling and Mode Competition in Multiple Anapole Systems
}

\author{
Valerio Mazzone, Juan Sebastian Totero Gongora and Andrea Fratalocchi * \\ PRIMALIGHT, Faculty of Electrical Engineering, Applied Mathematics and Computational Science, \\ King Abdullah University of Science and Technology, Thuwal 23955-6900, Saudi Arabia; \\ valerio.mazzone@kaust.edu.sa (V.M.); js.totero@kaust.edu.sa (J.S.T.G.) \\ * Correspondence: andrea.fratalocchi@kaust.edu.sa; Tel.: +966-(2)-808-0348
}

Academic Editor: Boris Malomed

Received: 17 April 2017; Accepted: 11 May 2017; Published: 24 May 2017

\begin{abstract}
All-dielectric metamaterials are a promising platform for the development of integrated photonics applications. In this work, we investigate the mutual coupling and interaction of an ensemble of anapole states in silicon nanoparticles. Anapoles are intriguing non-radiating states originated by the superposition of internal multipole components which cancel each other in the far-field. While the properties of anapole states in single nanoparticles have been extensively studied, the mutual interaction and coupling of several anapole states have not been characterized. By combining first-principles simulations and analytical results, we demonstrate the transferring of anapole states across an ensemble of nanoparticles, opening to the development of advanced integrated devices and robust waveguides relying on non-radiating modes.
\end{abstract}

Keywords: anapole; silicon nanoparticles; integrated photonics; FDTD; near-field coupling

\section{Introduction}

Dielectric nanostructures at optical frequencies are characterized by an extremely complex landscape of interacting resonant states. By finely tuning the material and geometrical properties of the nanostructures, it is possible to engineer advanced functionalities and applications such as, e.g., anti-reflection surfaces [1] and integrated waveguides based on chains of nanoparticles [2]. One of the most fascinating manifestations of multi-mode interaction in dielectric nanoparticles is the formation of radiationless states known as anapoles. These states have recently been demonstrated in silicon nanoparticles [3]. Anapoles are characterized by a strong reduction of the scattering from the nanoparticle at the anapole wavelength, which acquires the character of a fully-cloaked structure $[4,5]$. The mechanism underlying the formation of anapole states is the superposition of internal multi-mode components of the nanoparticle, which cancel each other in the far-field and which produce a radiation pattern confined to the near-field. As a result, anapole states are not the result of any resonant process (as in the case of dark resonances), but their origin lies entirely in modal competition and superposition [6]. While the theoretical description of anapole states in single dielectric nanoparticles is well established, the mutual coupling and interaction along a chain of anapole nanoparticles have yet to be investigated. Arrays of nanoparticles have been the subject of intense study, starting from the remarkable guiding properties demonstrated in plasmonic nanochains $[7,8]$. To mitigate the effect of metal losses, researchers have focused on all-dielectric solutions based on high refractive index nanoparticles [9]. As discussed in [10], the guiding properties of nanoparticle arrays can be strongly enhanced by minimizing the electromagnetic scattering from each nanoparticle in the array. As a result, anapole states could represent-in principle—a perfect candidate for efficient integrated waveguides based on silicon nanoparticles. However, due to their intrinsic non-resonant nature, anapoles should not manifest mutual coupling (as would be expected in the case of standard resonant 
states). Therefore, it should not be possible to transfer radiationless states among closely-packed dielectric structures. As recently demonstrated in two-dimensional cylinders, however, anapole states can be re-interpreted as the result of Fano interference between two or more, overlapping resonant states in the proximity of the anapole wavelength [11]. If such analysis could be extended to the three-dimensional case, it would be possible to describe and tailor the mutual coupling of an ensemble of invisible nanostructures. In this work, we address this problem by combining first-principles simulations and analytical theory, showing how the anapole state can be effectively transferred among distinct nanoparticles. Our results play a key role in the development of integrated optical circuitry based on non-radiating states (e.g., anapole-based wave-guides). Due to the near-field confinement produced by the anapole excitation and the suppression of the scattered field, wave-guides based on non-radiating states are extremely robust against physical bending and splitting, opening to the realization of high-density optical circuitry entirely based on silicon.

\section{Results}

\subsection{Ab-Initio Analysis of Multiple Anapole Systems}

We performed finite-differences time-domain (FDTD) analysis by considering an ensemble of three-dimensional anapole nanoparticles, each composed of a silicon nanodisk of radius $R=150 \mathrm{~nm}$ and height $h=50 \mathrm{~nm}$. Each nanodisk is aligned with the $z$-axis, and it is illuminated by an $E_{x}$ polarized plane wave propagating along the cylinder axis. In order to characterize the mutual coupling of closely-packed anapole nanoparticles, we consider a system of two slightly displaced nanodisks (see Figure 1a). The relative displacement of the identical nanoparticles is described by a centre-to-centre distance $d$ and by a rotation angle $\alpha$ measured from the $x$-axis (Figure 1a-inset).

The anapole wavelength $\lambda_{\text {an }}$ is identified by analysing the scattering cross-section $C_{\text {sca }}$ of the isolated structures, as reported in Figure $1 \mathrm{~b}$ (blue line). In the proposed configuration, the anapole state corresponds to $\lambda_{\mathrm{an}}=568 \mathrm{~nm}$ (green dashed line). Despite the strong reduction of the scattering cross-section, the anapole state is associated with a strong enhancement of the electric field inside the nanodisk. The field enhancement is measured by integrating the electric intensity inside the resonator (Figure $2 b$, dashed orange line), which exhibits a strong peak at the anapole wavelength $\lambda_{\text {an }}$. The strong field enhancement associated to the anapole state is a counter-intuitive feature of the non-radiating state, and it has recently been exploited to amplify light-matter processes in semiconductor nanostructures [12,13]. To characterize the mutual coupling between anapole states, we performed a set of simulations for different rotation angles $\alpha$ and distances $d$, whose results are reported in Figure 1c,d. In our numerical experiments, we selectively excited one of the two nanoparticles at the anapole wavelength $\lambda_{\text {an }}$, and we measured the steady-state electric field intensity $|\mathbf{E}|$ in the second resonator. In terms of angular displacement $\alpha$, the anapole coupling is characterized by a symmetric dipolar profile (Figure 1c). The near-field coupling is maximum in the direction of the three-lobe profile of the anapole state $(\alpha=0,2 \pi)$, while it is negligible in the orthogonal direction $(\alpha=\pi / 2,3 \pi / 2)$. Conversely, in terms of the mutual distance $d$, the anapole coupling exhibits a more complex profile, with a sharp $20 \%$ reduction in less than $150 \mathrm{~nm}$ total displacement (Figure 1d). Interestingly, the spatial decay of the anapole coupling does not follow a power law decay, as would be expected from near-field dipolar states. To verify this, we compared the anapole coupling distribution from Figure $1 \mathrm{c}, \mathrm{d}$ to the scattered field of an isolated nanoparticle (see Supplementary Figure S1). Even in the case of an isolated nanoparticle, the scattered field is mostly dipolar, as multipole-components are negligible at the anapole wavelength [3]. The dipolar angular distribution-associated with a complex decay profile—can be considered as a first signature of a complex modal interaction between closely-packed anapole nanoparticles. 


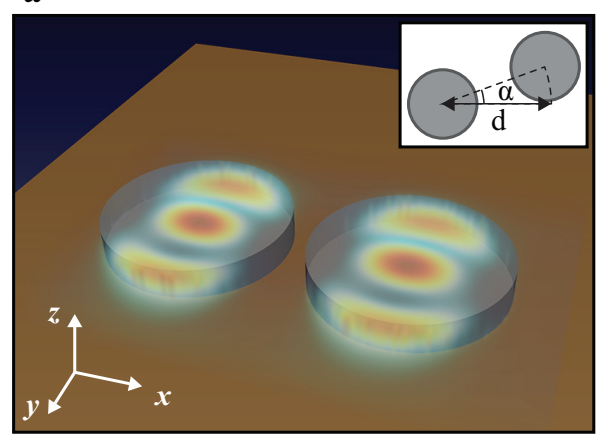

c

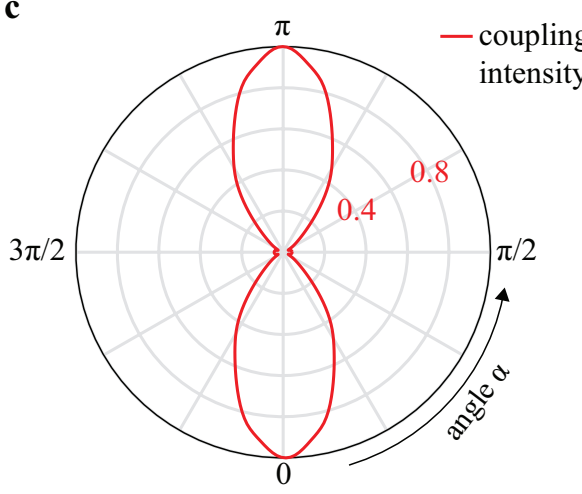

b
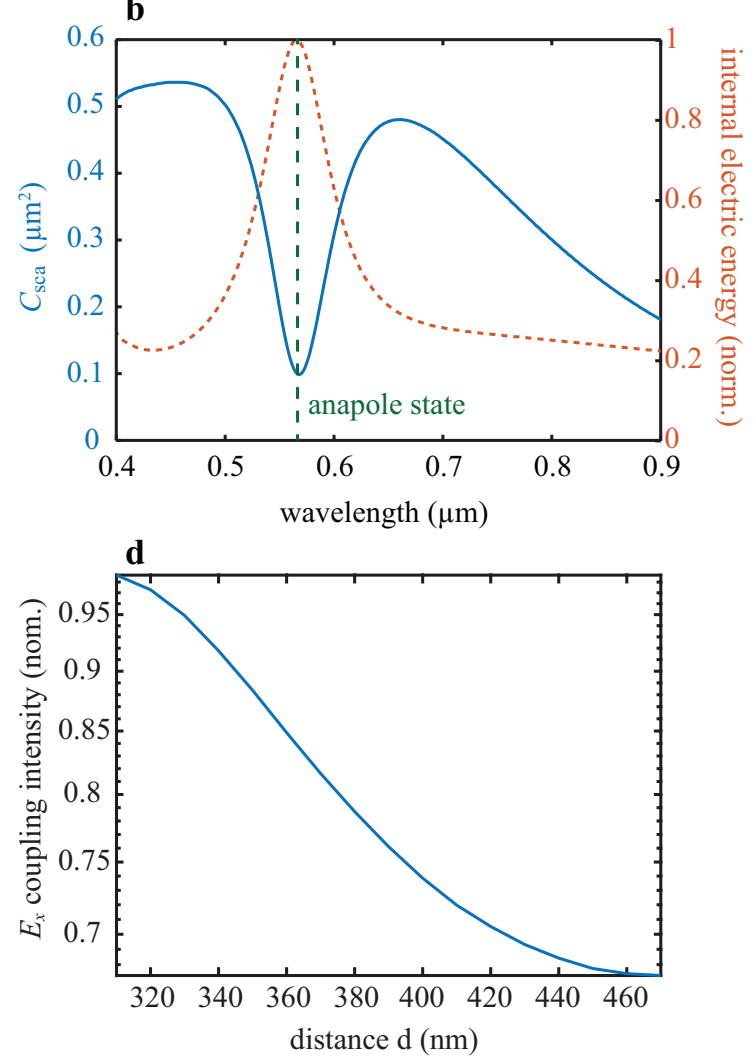

Figure 1. Mutual coupling of non-radiating anapole states. (a) Near-field coupling between two silicon nanodisks ( $n=3.5$ ) excited at the anapole wavelength $\lambda_{\text {an }}=568 \mathrm{~nm}$. (inset) The dielectric resonators are mutually displaced by a centre-to-centre distance $d$ and by an angle $\alpha$; (b) Scattering cross-section $C_{\text {sca }}$ (blue line) and internal electric energy (orange dotted line) as a function of the incident wavelength $\lambda$. The anapole state (green-dashed line) is characterized by the simultaneous suppression of the scattering cross-section, and by a strong enhancement of the internal field intensity; (c) Coupled electric energy as a function of the rotation angle $\alpha(d=450 \mathrm{~nm})$. The mutual coupling is maximum at $\alpha=0, \pi$ and negligible at $\alpha=\pi / 2,3 \pi / 2 ;(\mathbf{d})$ Coupled electric energy as a function of the mutual distance $d$. The results correspond to the angular condition of maximum scattering $\alpha=2 \pi$.

\subsection{Fano-Feshbach Analysis of the Internal Modes}

The counter-intuitive coupling properties of anapole-anapole systems can be explained by analysing the internal resonances of the system. The resonant properties of the single nanostructures can be extracted from the integrated density of states (DOS) $\rho(\lambda)$, which can be directly computed from FDTD simulations [14]. The integrated DOS is defined as:

$$
\rho(\lambda)=\int \mathrm{d} \mathbf{x} \rho(\mathbf{x}, \lambda)
$$

where $\rho(\mathbf{x}, \lambda)$ is the local DOS, which is a function of the spatial position inside the resonator. In the FDTD framework, the local DOS corresponds to the spectral response to a single pulse excitation, and it can be defined separately for each component of the electromagnetic fields. For a generic component $E_{j}$ of the electric field, as an example, the integrated DOS reads: 


$$
\rho_{E_{j}}(\omega)=\int \mathrm{d} \mathbf{x}\left|\mathcal{F}\left\{E_{j}(\mathbf{x}, t)\right\}\right|^{2},
$$

where $\mathcal{F}[\cdots]$ stands for Fourier transform and $E_{j}(\mathbf{x}, t)$ is the electric field along the $j$-th direction $(j=x, y, z)$. An analogue definition holds for the magnetic field components $H_{j}(\mathbf{x}, t)$.

As can be easily verified by solving Maxwell's equations in cylindrical coordinates $(r, \phi)$, the response from the anapole resonator can be fully represented in terms of the $E_{z}$ and $H_{z}$ field components [15]. In Figure 2, we report the integrated DOS for the (a) $H_{z}$ and (b) $E_{z}$ field components. The integrated DOS are computed by considering a single pulse excitation centred at $\lambda=700 \mathrm{~nm}$, and the spectral responses are normalized to the source spectrum. Interestingly, the transverse electric (TE) spectrum exhibits a strong peak exactly at the anapole wavelength (red vertical line), while the transverse magnetic (TM) spectrum shows only a slight enhancement at the anapole frequency (Figure 2b).
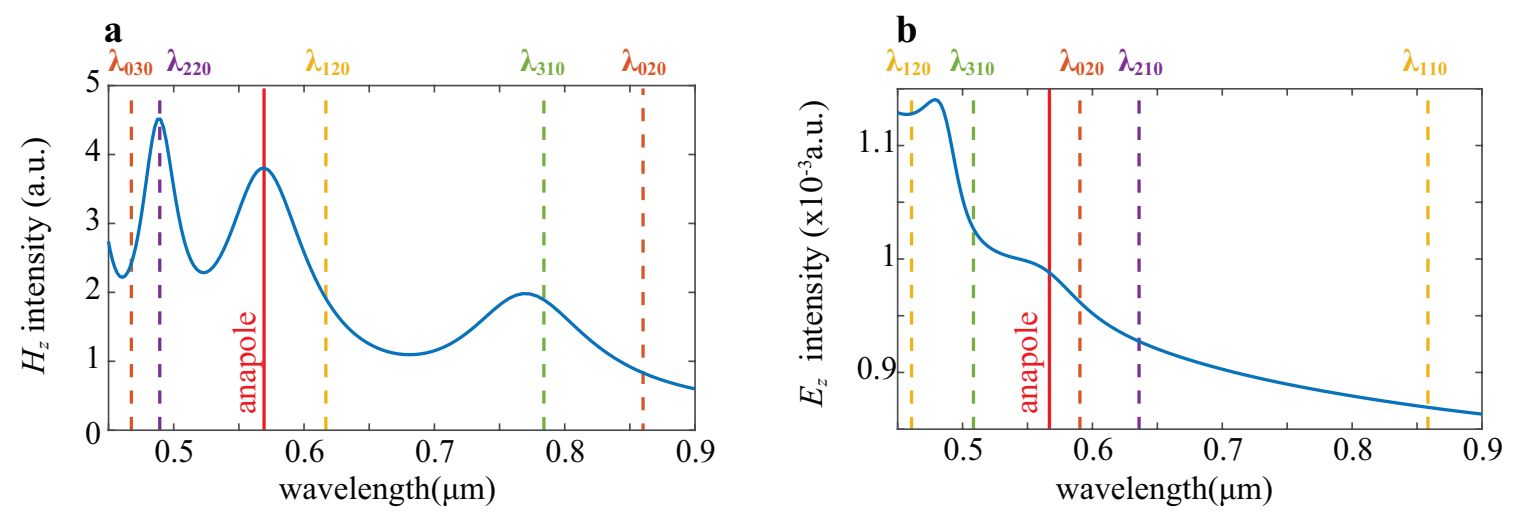

Figure 2. Local density of states and interacting resonant modes. (a,b) Local density of state for the (a) $H_{z}$ and (b) $E_{z}$ field components, corresponding to the transverse electric (TE) and transverse magnetic (TM) modes of the silicon nanostructure, respectively. The resonant wavelengths (vertical dashed lines) in both configurations are computed by means of Equation (4).

Further insights on the resonant properties of the anapole state can be obtained by decomposing the electromagnetic fields into a set of orthogonal eigenmodes. By definition, however, the silicon resonator represents an open cavity, and the definition of a set of orthogonal resonator modes is a challenging task $[16,17]$. As recently shown in [11], a possible way to circumvent such difficulties is the introduction of a Fano-Feshbach partitioning of the system [18]. In a nutshell, the Fano-Feshbach partitioning consists of the mathematical splitting of the total system into two orthogonal eigenspaces, corresponding to the resonator and environment regions. Maxwell's equations are rewritten in the partitioned subspaces, providing a rigorous description of light-matter interaction in open resonators. For a cylinder aligned along the $z$-axis, the Fano-Feshbach internal resonances for the fields $H_{z}$ and $E_{z}$ correspond to the TE and TM modes of a perfect electric conductor (PEC) cavity [19]. They are expressed as:

$$
\left\{\begin{array}{l}
\mathrm{TE}_{m p q}=N_{m p q} J_{m}\left(\frac{\chi_{m p}^{\prime}}{R} r\right) \sin \left(\frac{q \pi}{h n}\right) \exp (i m \phi) \\
\mathrm{TM}_{m p q}=M_{m p q} J_{m}\left(\frac{\chi_{m p}}{R} r\right) \cos \left(\frac{q \pi}{h n}\right) \exp (i m \phi)
\end{array}\right.
$$

where $J_{m}$ is a Bessel function of the first-kind of order $m ; N_{m p q}, M_{m p q}$ are normalization constants; and where $\chi_{m p}, \chi_{m p}^{\prime}$ denote the $p$-th zero of the Bessel function $J_{m}$ and its derivative $J_{m}^{\prime}=\partial J_{m} / \partial \rho$, respectively. The resonance frequencies of the internal modes are defined as: 


$$
\left\{\begin{array}{l}
\omega_{m p q}^{2}=\frac{c^{2}}{n^{2}}\left[\left(\frac{q \pi}{h n}\right)^{2}+\left(\frac{\chi_{m p}^{\prime}}{R}\right)\right] \quad \text { TE modes } \\
\omega_{m p q}^{2}=\frac{c^{2}}{n^{2}}\left[\left(\frac{q \pi}{h n}\right)^{2}+\left(\frac{\chi_{m p}}{R}\right)\right] \quad \text { TM modes. }
\end{array}\right.
$$

The choice of PEC boundary conditions for the internal modes is not fixed, as it is only dictated by the mathematical partitioning scheme adopted. As thoroughly discussed in $[11,18,20]$, they can be easily exchanged with perfect magnetic conductor (PMC) boundary conditions, which are usually employed to analyse high refractive index nanoparticles [21,22]. A preliminary analysis of the system in terms of Fano-Feshbach internal resonances allows us to unveil some fundamental properties of the anapole excitation. By solving Equation (4), we computed the Fano-Feshbach resonant frequencies $\omega_{m p q}$ of the system, which are reported as sets of dashed vertical lines on Figure 2. Interestingly, the anapole state is generated in the proximity of a few orthogonal resonances. Among all the available candidates, the characteristic three-lobes mode profile of the anapole state can be obtained by superimposing two TM modes (Figure 3): a cylindrically symmetric $\mathrm{TM}_{020}$ and a quadrupolar $\mathrm{TM}_{210}$. Remarkably, these modes constitute the three-dimensional version of the eigenmodes composing the anapole state in two-dimensional cylinders (cfr. Figure 3 of [11]).

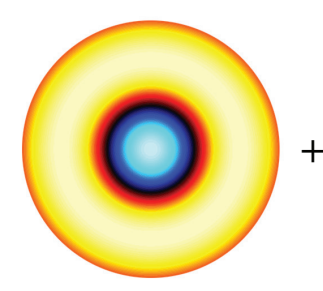

$\mathrm{TM}_{020}$

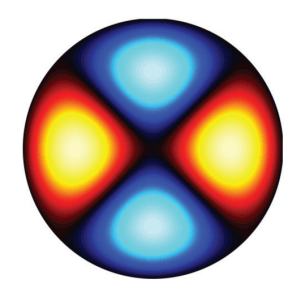

$\mathrm{TM}_{210}$

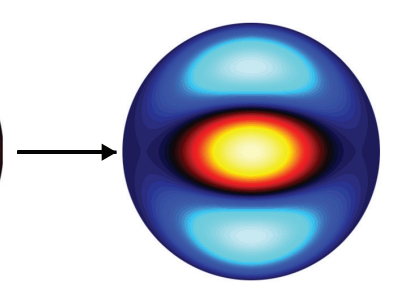

anapole

Figure 3. Fano-Feshbach partitioning of the anapole state. The characteristic mode-profile of the anapole state is originated by the superposition of a cylindrically symmetric $\mathrm{TM}_{020}$ and a quadrupolar $\mathrm{TM}_{210}$.

\section{Discussion and Conclusions}

By combining FDTD simulations and analytical theory, we have shown how anapole states can be coupled and transferred among closely-packed nanoparticles. This result-which recalls the coupling properties of standard resonant modes-is at first counter-intuitive due to the non-resonant character of the anapole state. However, as can be demonstrated by performing a Fano-Feshbach partitioning of the anapole resonator, the three-dimensional state is characterized by the superposition of several distinct resonances of the system, which collectively produce a scattering-suppression state at the anapole wavelength and which mutually couple among the ensemble of nanoparticles. The Fano-Feshbach analysis of three-dimensional anapole states-including a detailed analysis of the scattered fields-goes beyond the scope of this work, and it will be the subject of a future specialized work on the topic.

The ability to control and transfer non-radiating states along optical circuitry, however, opens to intriguing possibilities in the development of advanced integrated photonics platforms. As shown in Figure 4, anapole nanoparticles can in fact be arranged in a compact nanochain with remarkable guiding properties. Due to the efficient near-field coupling between adjacent nanoparticles, the nanochain can support guided modes which propagate without radiative losses at distances of several $\mu \mathrm{m}$. In these simulations, we selectively excited the first anapole state in the nanochain (not shown in the figure) and we characterized the propagation of energy along the chain. Such excitation can be achieved experimentally, as single anapole nanoparticles can be excited by 
means of a near-field scanning optical microscopy (NSOM) setup [3]. As an appealing alternative, anapole-based waveguides could be combined with integrated nanolasers emitting at the anapole frequency [13].

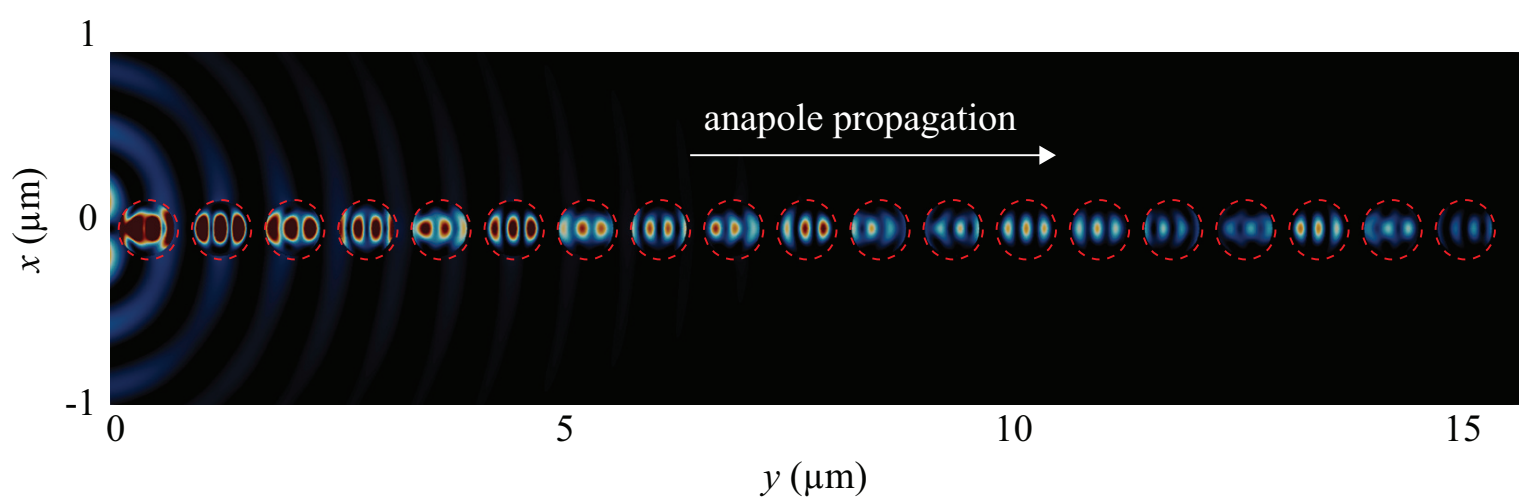

Figure 4. Anapole nanochain. Steady-state electromagnetic energy distribution along a chain of anapole nanoparticles. The centre-to-centre distance is $d=400 \mathrm{~nm}$. The external excitation is restricted to the first anapole of the chain (not included in the panel).

As the anapole states are tightly confined in the near-field, optical nano-circuitry based on non-radiating modes is extremely robust to bending and splitting, as shown in Figure 5. In standard photonics applications, wave-guide deformation produces significant radiation losses, in particular when considering 90-degree bends and turns [23]. Conversely, in the case of an anapole nanochain, the near-field properties of the non-radiating state allow for efficient transmission of the guided mode across deformations and bends, such as in the case of wave-guide splitting (Figure 5a) or 90-degree bending and re-routing (Figure 5b). These illustrative examples strongly suggest the possibility of integrating anapole nanoparticles with state-of-the-art integrated photonics applications.
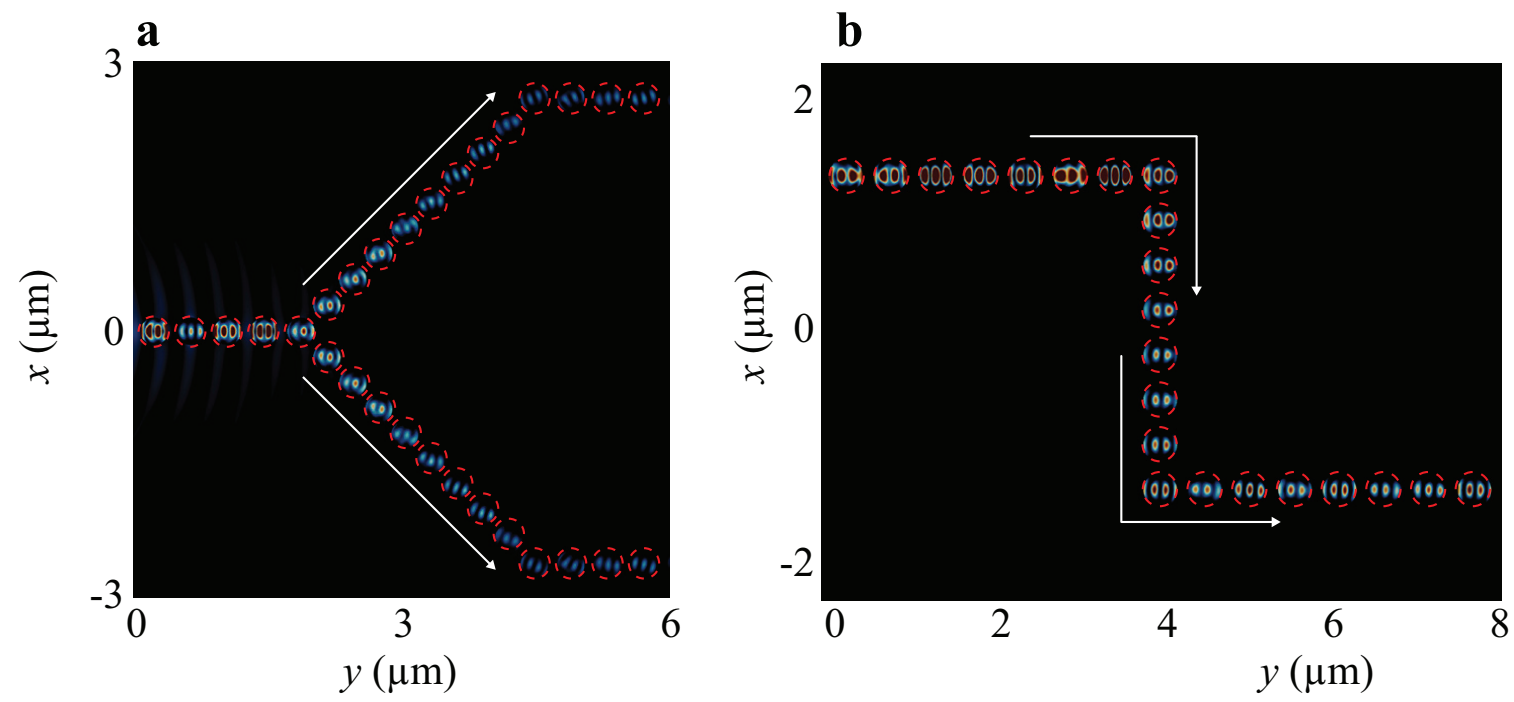

Figure 5. Robust sub-wavelength guiding via near-field transfer of anapole states. Due to the near-field confinement produced by the anapole state, the anapole nanochain is robust against bending and splitting of the integrated wave-guide. This opens to the realization of (a) integrated splitters and (b) 90-degree bends without introducing radiation losses. 


\section{Materials and Methods}

\subsection{FDTD Simulations}

We performed fully-dispersive three-dimensional FDTD simulations using our home-made simulator NANOCPP [24-29]. In our simulations, the computational domain was organized as follows: the $\mathrm{z}$-aligned nanodisks were placed at the centre of a $2 \mu \mathrm{m} \times 2 \mu \mathrm{m} \times 1 \mu \mathrm{m}$ box, with uniaxial perfectly matched layer (UPML) boundary conditions emulating an open system [30]. The numerical resolution was set as $\Delta x=2 \mathrm{~nm}$, corresponding to 81 points per internal wavelength at the anapole frequency $\lambda_{\mathrm{an}}$. The system was illuminated by plane wave, implemented according to the total-field scattered-field (TFSF) formalism [31]. The scattering cross-section (Figure 1b) was computed by integrating the Poynting vector along a three-dimensional surface surrounding the objects and entirely placed in the scattered-field region of the TFSF. In order to characterize the anapole coupling among distinct nanoparticles, we included one of the resonators in the total-field region of the TFSF, while the other resonators were placed in the scattered-field region.

Supplementary Materials: The following are available online at http:/ /www.mdpi.com/2076-3417/7/6/542/s1, Figure S1: Scattered field from an isolated anapole state.

Acknowledgments: This research is supported by KAUST (Award No. OSR-2016-CRG5-2995). For the computer time, we have used the resources of the KAUST Supercomputing Laboratory and the Redragon cluster of the PRIMALIGHT group.

Author Contributions: V.M. and J.S.T.G. performed first principle parallel simulations and analytical modelling. A.F. supervised the research. All authors contributed to the analysis of data. All authors contributed equally to manuscript preparation.

Conflicts of Interest: The authors declare no conflict of interest.

Abbreviations
$\begin{array}{ll}\text { The following abbreviations are used in this manuscript: } \\ \text { FDTD } & \text { Finite-Differences Time-Domain } \\ \text { DOS } & \text { Density Of States } \\ \text { UPML } & \text { Uniaxial Perfectly Matched Layer } \\ \text { TFSF } & \text { Total-Field Scattered-field } \\ \text { TM } & \text { Transverse Magnetic } \\ \text { TE } & \text { Transverse Electric } \\ \text { PEC } & \text { Perfect Electric Conductor } \\ \text { PMC } & \text { Perfect Magnetic Conductor } \\ \text { NSOM } & \text { Near-field scanning optical microscopy }\end{array}$

\section{References}

1. Wang, K.X.; Yu, Z.; Sandhu, S.; Liu, V.; Fan, S. Condition for perfect antireflection by optical resonance at material interface. Optica 2014, 1, 388-395.

2. Liu, W.; Miroshnichenko, A.E.; Neshev, D.N.; Kivshar, Y.S. Broadband Unidirectional Scattering by Magneto-Electric Core-Shell Nanoparticles. ACS Nano 2012, 6, 5489-5497.

3. Miroshnichenko, A.E.; Evlyukhin, A.B.; Yu, Y.F.; Bakker, R.M.; Chipouline, A.; Kuznetsov, A.I.; Luk'yanchuk, B.; Chichkov, B.N.; Kivshar, Y.S. Nonradiating anapole modes in dielectric nanoparticles. Nat. Commun. 2015, 6, 8069.

4. Liu, W.; Zhang, J.; Lei, B.; Hu, H.; Miroshnichenko, A.E. Invisible nanowires with interfering electric and toroidal dipoles. Optics Lett. 2015, 40, 2293-2296.

5. Luk'yanchuk, B.; Paniagua-Domínguez, R.; Kuznetsov, A.I.; Miroshnichenko, A.E.; Kivshar, Y.S. Suppression of scattering for small dielectric particles: anapole mode and invisibility. Philos. Trans. R. Soc. Lond. A Math. Phys. Eng. Sci. 2017, 375, 20160069. 
6. Wei, L.; Xi, Z.; Bhattacharya, N.; Urbach, H.P. Excitation of the radiationless anapole mode. Optica 2016, 3, 799-802.

7. Brongersma, M.L.; Hartman, J.W.; Atwater, H.A. Electromagnetic energy transfer and switching in nanoparticle chain arrays below the diffraction limit. Phys. Rev. B 2000, 62, R16356-R16359.

8. Totero Gongora, J.S.; Fratalocchi, A. Harnessing Disorder at the Nanoscale. In Encyclopedia of Nanotechnology; Bhushan, B., Ed.; Springer Netherlands: Heidelberg, Germany, 2015; pp. 1-13.

9. Bakker, R.M.; Yu, Y.F.; Paniagua-Domínguez, R.; Luk'yanchuk, B.; Kuznetsov, A. Silicon Nanoparticles for Waveguiding. Frontiers in Optics; Optical Society of America: Washington, DC, USA, 2015; p. FM1B.2.

10. Evlyukhin, A.B.; Reinhardt, C.; Seidel, A.; Luk'yanchuk, B.S.; Chichkov, B.N. Optical response features of Si-nanoparticle arrays. Phys. Rev. B 2010, 82, 045404.

11. Gongora, J.S.T.; Favraud, G.; Fratalocchi, A. Fundamental and high-order anapoles in all-dielectric metamaterials via Fano-Feshbach modes competition. Nanotechnology 2017, 28, 104001.

12. Grinblat, G.; Li, Y.; Nielsen, M.P.; Oulton, R.F.; Maier, S.A. Efficient Third Harmonic Generation and Nonlinear Subwavelength Imaging at a Higher-Order Anapole Mode in a Single Germanium Nanodisk. ACS Nano 2017, 11, 953-960.

13. Totero Gongora, J.S.; Miroshnichenko, A.E.; Kivshar, Y.S.; Fratalocchi, A. Anapole nanolasers for mode-locking and ultrafast pulse generation. Nat. Commun. 2017, in press.

14. Taflove, A.; Oskooi, A.; Johnson, S.G. Advances in FDTD Computational Electrodynamics: Photonics and Nanotechnology; Artech House Antennas and Propagation Series; Artech House: Norwood, MA, USA, 2013.

15. Yariv, A.; Yeh, P. Photonics: Optical Electronics in Modern Communications (The Oxford Series in Electrical and Computer Engineering); Oxford University Press, Inc.: New York, NY, USA, 2006.

16. Türeci, H.E.; Ge, L.; Rotter, S.; Stone, A.D. Strong Interactions in Multimode Random Lasers. Science 2008, 320, 643-646.

17. Kristensen, P.T.; Hughes, S. Modes and Mode Volumes of Leaky Optical Cavities and Plasmonic Nanoresonators. ACS Photonics 2014, 1, 2-10.

18. Viviescas, C.; Hackenbroich, G. Field quantization for open optical cavities. Phys. Rev. A 2003, 67, 013805.

19. Jin, J.M. Theory and Computation of Electromagnetic Fields; John Wiley \& Sons: Hoboken, NJ, USA, 2011; Google-Books-ID: D6SqmxJVV5wC.

20. Viviescas, C.; Hackenbroich, G. Quantum theory of multimode fields: Applications to optical resonators. J. Opt. B Quantum Semiclass. Opt. 2004, 6, 211.

21. Van Bladel, J. Radiation in Free Space. In Electromagnetic Fields; Wiley-IEEE Press: Hoboken, NJ, USA, 2007; pp. 277-356.

22. Tribelsky, M.I.; Miroshnichenko, A.E. Giant in-particle field concentration and Fano resonances at light scattering by high-refractive-index particles. Phys. Rev. A 2016, 93, 053837.

23. Cherchi, M.; Ylinen, S.; Harjanne, M.; Kapulainen, M.; Aalto, T. Dramatic size reduction of waveguide bends on a micron-scale silicon photonic platform. Opt. Express 2013, 21, 17814.

24. Gentilini, S.; Fratalocchi, A.; Angelani, L.; Ruocco, G.; Conti, C. Ultrashort pulse propagation and the Anderson localization. Opt. Lett. 2009, 34, 130-132.

25. Crosta, M.; Fratalocchi, A.; Trillo, S. Bistability and instability of dark-antidark solitons in the cubic-quintic nonlinear Schrödinger equation. Phys. Rev. A 2011, 84, 063809.

26. Huang, J.; Liu, C.; Zhu, Y.; Masala, S.; Alarousu, E.; Han, Y.; Fratalocchi, A. Harnessing structural darkness in the visible and infrared wavelengths for a new source of light. Nat. Nanotechnol. 2016, 11, 60-66.

27. Totero Gongora, J.S.; Miroshnichenko, A.E.; Kivshar, Y.S.; Fratalocchi, A. Energy equipartition and unidirectional emission in a spaser nanolaser. Laser Photonics Rev. 2016, 10, 432-440.

28. Labelle, A.J.; Bonifazi, M.; Tian, Y.; Wong, C.; Hoogland, S.; Favraud, G.; Walters, G.; Sutherland, B.; Liu, M.; Li, J.; et al. Broadband Epsilon-near-Zero Reflectors Enhance the Quantum Efficiency of Thin Solar Cells at Visible and Infrared Wavelengths. ACS Appl. Mater. Interfaces 2017, 9, 5556-5565.

29. Galinski, H.; Favraud, G.; Dong, H.; Totero Gongora, J.S.; Favaro, G.; Döbeli, M.; Spolenak, R.; Fratalocchi, A.; Capasso, F. Scalable, ultra-resistant structural colors based on network metamaterials. Light Sci. Appl. 2017, 6, e16233. 
30. Berenger, J.P. A Perfectly Matched Layer for the Absorption of Electromagnetic-Waves. J. Comput. Phys. 1994, 114, 185-200.

31. Taflove, A.; Hagness, S.C. Computational Electrodynamics: The Finite-Difference Time-Domain Method, 3rd ed.; Artech House Antennas and Propagation Library, Artech House: Boston, MA, USA, 2005. 